\title{
Dynamic volume holography and optical information processing by Raman scattering
}

\author{
I.Y. Dodin*, N.J. Fisch \\ Department of Astrophysical Sciences, Princeton Plasma Physics Laboratory, Princeton University, Princeton, NJ 08543, USA
}

Received 29 August 2002; received in revised form 29 October 2002; accepted 29 October 2002

\begin{abstract}
A method of producing holograms of three-dimensional (3D) optical pulses is proposed. It is shown that both the amplitude and the phase profile of 3D optical pulse can be stored in dynamic perturbations of a Raman medium, such as plasma. By employing Raman scattering in a nonlinear medium, information carried by a laser pulse can be captured in the form of a slowly propagating low-frequency wave that persists for a time large compared with the pulse duration. If such a hologram is then probed with a short laser pulse, the information stored in the medium can be retrieved in a second scattered electromagnetic wave. The recording and retrieving processes can conserve robustly the pulse shape, thus enabling the recording and retrieving with fidelity of information stored in optical signals. While storing or reading the pulse structure, the optical information can be processed as an analog or digital signal, which allows simultaneous transformation of $3 \mathrm{D}$ continuous images or computing discrete arrays of binary data. By adjusting the phase fronts of the reference pulses, one can also perform focusing, redirecting, and other types of transformation of the output pulses.
\end{abstract}

(c) 2002 Elsevier Science B.V. All rights reserved.

PACS: 42.40.Ht; 42.65.Dr; 52.35.Fp; 52.38.Bv

Keywords: Holography; Raman scattering; 3D pulses

\section{Introduction}

Holographic methods of recording and retrieving wave fields were first proposed about 50 years ago $[1,2]$. Conventional techniques of holography are based on recording both the amplitude and the

\footnotetext{
${ }^{*}$ Corresponding author. Tel.: +1-609-9334935; fax: +1-6092432662.

E-mail address: idodin@pppl.gov (I.Y. Dodin).
}

phase information of an input light wave into static perturbations of the refraction coefficient of photoemulsion. The perturbations are driven by the static beating of an input signal and a reference recording wave. After the pattern is imprinted into photoemulsion, it can be retrieved by scanning the hologram with another reference wave of the same frequency. In such a static hologram, the information is "carved" in static perturbations of the medium, so that only one holographic image can be stored within a given sample. 
Transient (dynamic) holograms [3-5] are produced by information and reference waves of slightly different frequencies, which store a holographic image in some low-frequency electrostatic wave resonant with the beating of the two electromagnetic signals. In strongly dissipative media, as the information wave field slowly changes in time, the hologram transforms quasistatically and reproduces the current interference pattern of the information and the reference waves at each moment of time. The invention of dynamic holography made high-speed processing of optical information possible and was employed in applications such as "real-time" image processing, optical computing, as well as optical mass storage and wavefront reversal [6]. In recent years these applications have stimulated research on materials with dynamic holographic capabilities, which is useful for increasing the degree of parallelism in manipulating optical data; multiplexing techniques, which are also useful in increasing storage capacity, have been devised [7].

Dynamic holography is applicable to dealing with relatively fast signals, but nevertheless it has severe limitations on information density in the information wave. The quasistatic electromagnetic wave profile, which contains the holographic image, needs to become settled in order for optical information to be recorded with fidelity (see, e.g., [8]). Thus, in principle, conventional dynamic holograms cannot resolve the longitudinal structure of the pulse.

To record the longitudinal structure of the information pulse in a medium, there exist at least two different opportunities. The first one, which was implemented in the experiments with electromagnetically induced transparency (EIT) $[9,10]$, is to let the whole pulse into the medium and then imprint its structure instantaneously on the whole length of the pulse by means of a reference signal coming from one side. In this case, substantial deceleration of the optical pulse is needed if the length of the pulse in vacuum exceeds the length of a given sample of the nonlinear medium ("a holographic plate"). Another constraint consists of strong anisotropy of the hologram providing that a reference signal imprints the image instantaneously, so that the displacement of the informa- tion pulse is negligible during the recording process.

An alternative approach of recording both transverse and longitudinal structures of optical pulses in a nonlinear medium was proposed in our previous work [11]. We showed that both recording and retrieving can be provided by probing the medium with short reference pulses that scan the longitudinal profiles of the information pulse and the hologram. Producing such static volume holograms of such kind was discussed, for example, in [12] with reference to a specific medium of a coupled-resonator optical waveguide. (In [12], the term "dynamic hologram" is used instead to emphasize that the proposed technique deals with recording the longitudinal structure of a pulse.) Contrary to the technique proposed for "stopping the light" in EIT experiments, this method does not necessarily require that the linear dispersion properties of the medium for the information pulse must differ drastically from those with vacuum.

In this paper, we propose a technique, which combines the advantages of both conventional dynamic and volume holography, and allows storing and processing of optical information by recording and retrieving transient three-dimensional (3D) holographic images in Raman media. The recording, storing and retrieving optical information is by means of the third wave with finite frequency. Further in this paper we term the proposed technique as "dynamic volume holography", or DVH. The image of the original pulse imprinted onto the shape of the low-frequency wave envelope is called then a dynamic hologram of the pulse. We show that DVH can, in principle, be realized in arbitrary Raman media (gases, fibers, plasmas), where beatings of high-frequency waves can resonantly excite low-frequency slowly propagating waves, such as, for example, sound or Langmuir waves, as long as these waves persist on time scales suitable for storing the optical information.

The paper is organized as follows. In Section 2, we set up the general problem and give the basic equations governing 1D nonlinear interaction of laser pulses during recording and retrieving. In Section 3, we discuss the features of recording and retrieving longitudinal structure of optical pulses 
in details. Section 4 is devoted to the applicability limits of the proposed approach. In Section 5, we complete the description of DVH by taking the transverse structure of the pulses into account and discuss the possibility of multichannel DVH, which allows dealing with multiplexed 1D data buses without spatial separation of the information channels. Further, in Section 6, we show that DVH allows more advanced processing of optical information, compared to the conventional dynamic holography. Processing can include various linear conversions of analog data, such as, for example, taking the derivatives of an optical pulse profile, or performing logical operations on digital signals. The difficulties one faces while implementing DVH in a nonideal Raman medium and possible applications of the proposed technique are discussed in Section 7. The summary of the main ideas is given in Section 8 .

\section{1D problem: basic equations}

In order to get the main idea of DVH, we first discuss the 1D problem of recording and retrieving longitudinal profiles of optical pulses. To proceed, consider the 1D interaction of information wave envelopes $a$ and $f$ and a reference pulse $b$, having frequencies $\omega_{a}, \omega_{f}, \omega_{b}$ and wavenumbers $k_{a}, k_{f}, k_{b}$ correspondingly, which satisfy the conditions of resonant interaction:

$\omega_{a}=\omega_{b}+\omega_{f}, \quad k_{a}=k_{b}+k_{f}$.

Three-wave interaction equations can be put in the form (see, e.g., [13])

$\partial_{t} a+V_{a} \partial_{z} a=\mu_{a} b f$

$\partial_{t} b+V_{b} \partial_{z} b=-\mu_{b} a f^{*}$

$\partial_{t} f+V_{f} \partial_{z} f=-\mu_{f} a b^{*}$,

where $V_{a}, V_{b}$, and $V_{f}$ are the group velocities of the corresponding waves. For further analysis, we require that the constant coefficients $\mu_{a}$ and $\mu_{f}$ are of equal signs, which we take positive for clarity. No limitations are imposed on the sign of $\mu_{b}$, which is taken positive only to match the notation of $[11,14,15]$. Consider the change of variable $z^{\prime}=z-V_{b} t$, which we further term shifting to the frame of reference moving together with the pulse $b$. In the new frame, Eqs. (2)-(4) are given by

$\partial_{t} a+\left(V_{a}-V_{b}\right) \partial_{z^{\prime}} a=\mu_{a} b f$,

$\partial_{t} b=-\mu_{b} a f^{*}$,

$\partial_{t} f+\left(V_{f}-V_{b}\right) \partial_{z^{\prime}} f=-\mu_{f} a b^{*}$.

Suppose now that the length $\sigma$ of the pulse $b$ is short enough to provide that, in the moving frame, the evolution of $a$ and $f$ is adequately described by the quasistatic approximation (see, e.g., [13]). Namely, we assume that the temporal derivatives of $a$ and $f$ are small compared to the terms containing spatial derivatives in Eqs. (5) and (7). In the moving frame, the temporal variations of $a$ and $f$ are caused by two factors. The first one is the variation in boundary conditions for the information pulses:

$a\left(z^{\prime} \rightarrow \pm \infty\right) \rightarrow a_{0}^{( \pm)}(t), \quad f\left(z^{\prime} \rightarrow \pm \infty\right) \rightarrow f_{0}^{( \pm)}(t)$

(for $b$, zero boundary conditions are assumed), which do not break the validity of the quasistatic approximation if $\Lambda \gg \sigma$, where we denote the minimal characteristic length of both pulses $a$ and $f$ with $\Lambda$. In addition, the temporal variations of the information pulses are caused by the evolution of the pulse $b$ :

$\partial_{t} a / a \sim \partial_{t} f / f \sim \partial_{t} b / b \sim \mu_{b} a f / b$,

so in order to neglect $\partial_{t}$ with respect to $\partial_{z^{\prime}}$ in Eqs. (5) and (7), one needs

$b\left|V_{a, f}-V_{b}\right| / \sigma \gg \mu_{b} a f$.

Thus, in order for quasistatic approximation to hold, the pulse $b$ must be not only short compared to the pulses $a$ and $f$, but be also strong enough and propagate at a speed sufficiently different (in its sign or absolute value) from the group velocities of the information pulses. Since the group velocities $V_{a, f}$ and $V_{b}$ are signed quantities, from condition (10) it follows that the quasistatic approximation is best hold for counter-propagating pulses.

It is convenient to switch to dimensionless notation by changing $a \rightarrow C_{a} a, b \rightarrow C_{b} b, f \rightarrow C_{f} f$, introduce dimensionless group velocities $u_{a, b, f}=$ 
$V_{a, b, f} /\left|V_{b}\right|$ and variables $\xi=z / Z_{0}, \tau=t / T_{0}$, and $\zeta=\xi-u_{b} \tau$, where

$$
\begin{aligned}
& C_{a, f}=C_{b} \sqrt{\frac{\mu_{a, f}}{\mu_{b}} \frac{V_{b}}{V_{b}-V_{a, f}}}, \\
& Z_{0}=C_{b}^{-1} \sqrt{\frac{\left(V_{b}-V_{a}\right)\left(V_{b}-V_{f}\right)}{\mu_{a} \mu_{f}}}, \\
& T_{0}=Z_{0} /\left|V_{b}\right|,
\end{aligned}
$$

and $C_{b}$ is arbitrary. (Therefore, the specific values of the pulse amplitudes are not important in the sense that the field dynamics for another amplitudes can be obtained by simple rescaling.) So, under the quasistatic approximation, Eqs. (5)-(7) take the dimensionless form [14]

$\partial_{\zeta} a=b f, \quad \partial_{\tau} b=-a f^{*}, \quad \partial_{\zeta} f=-a b^{*}$.

From Eqs. (12), one can derive the ManleyRowe relation for the waves $a$ and $f:|a(\zeta, \tau)|^{2}+$ $|f(\zeta, \tau)|^{2}=\left|N_{0}(\tau)\right|^{2}$. Thus, if the phase $\theta$ of the wave envelope $b(\zeta, \tau)=B(\zeta, \tau) \mathrm{e}^{\mathrm{i} \theta}$ can be treated as constant, the wave envelopes are given by $[11,13,14]$

$$
\begin{aligned}
& a=N_{0} \cos \left(U / 2+\phi_{0}\right), \\
& f=-\mathrm{e}^{-\mathrm{i} \theta} N_{0} \sin \left(U / 2+\phi_{0}\right),
\end{aligned}
$$

where $U(\zeta, \tau)=2 \int_{-\infty}^{\zeta} B\left(\zeta^{\prime}, \tau\right) \mathrm{d} \zeta^{\prime}$ satisfies the sinGordon equation $\partial_{\zeta \tau}^{2} U=\sin \left(U+2 \phi_{0}\right)$.

\section{Recording and retrieving 1D dynamic volume holograms}

One-dimensional DVH of an optical wave envelope $a_{\text {in }}(\xi, \tau)=A_{\text {in }}^{(0)}\left(\zeta_{a}=\xi-u_{a} \tau\right)$ recorded by a reference laser pulse $b_{\text {rec }}(\zeta, \tau)=B_{\text {rec }}(\zeta, \tau) \mathrm{e}^{\mathrm{i} \theta_{\text {rec }}}$ represents a copy of the longitudinal structure of the pulse $A_{\mathrm{in}}^{(0)}$ in a spatial profile of lowfrequency wave envelope $f$, which is produced by the beating of the two electromagnetic waves (Fig. 1). Assume that dissipation of $F_{0}$ is negligible on the timescales of interest. At low power of the pulses, when each of the two light waves may be treated as given (the exact conditions will be discussed in Section 4), the imprinted

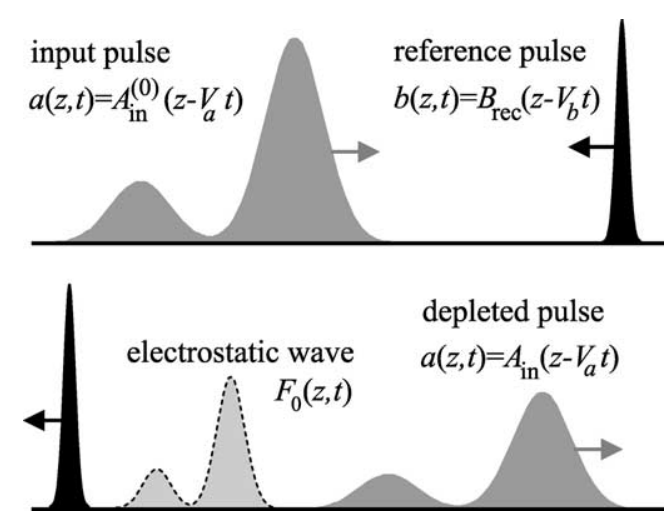

Fig. 1. Conceptual scheme of recording of the longitudinal structure of 1D optical pulse in Raman media. While scanning with a reference pulse $B_{\text {rec }}$, the longitudinal structure of the optical pulse $A_{\text {in }}^{(0)}$ is copied into the profile of electrostatic wave $F_{0}\left(\zeta_{f}\right) \propto A_{\text {in }}^{(0)}\left(\Upsilon_{\zeta_{f}}\right)$ (called a dynamic volume hologram). For counter-propagating optical pulses in boundless medium, the compression factor is $\Upsilon \approx 2$.

hologram is proportional to the convolution of the optical pulses:

$$
\begin{aligned}
f(\xi, \tau)= & F_{0}\left(\zeta_{f}=\xi-u_{f} \tau, \tau\right), \\
F_{0}\left(\zeta_{f}\right)=- & \int_{-\infty}^{+\infty} A_{\text {in }}^{(0)}\left(\zeta_{f}-\left(u_{a}-u_{f}\right) \zeta^{\prime}\right) \\
& B_{\text {rec }}\left(\zeta^{\prime}-\zeta_{f} /\left(u_{b}-u_{f}\right)\right) \mathrm{d} \zeta^{\prime},
\end{aligned}
$$

$\left(F_{0}\left(\zeta_{f}\right)=F_{0}\left(\zeta_{f}, \tau \rightarrow \infty\right)\right)$ as follows from Eq. (4). Assuming $B_{\text {rec }}$ short compared to $A_{\text {in }}^{(0)}$, i.e., $B_{\text {rec }}(\zeta)=\epsilon_{\text {rec }} \delta(\zeta)$, one gets

$$
\begin{aligned}
& F_{0}\left(\zeta_{f}\right)=-\mathrm{e}^{-\mathrm{i} \theta_{\mathrm{rec}}} \epsilon_{\mathrm{rec}} A_{\mathrm{in}}^{(0)}\left(\Upsilon \zeta_{f}\right), \\
& \Upsilon=\frac{u_{b}-u_{a}}{u_{b}-u_{f}}
\end{aligned}
$$

From Eqs. (15), one can see that the optical information is copied into the low-frequency wave $f$, which has the phase and the amplitude exactly the same than those of the original optical pulse, though its shape is $\Upsilon$-times compressed along the axis of pulse propagation. For the interaction of counter-propagating pulses, the compression factor is $\Upsilon \approx 2$, since $u_{a} \approx-u_{b} \gg u_{f}$.

It is important to emphasise that, contrary to the experiments with EIT $[9,10]$, in DVH the compression factor varies depending on the velocity of the reference signal, though the nonlinear interaction itself may have no influence on the 
dispersion properties of the information pulse. For example, compression with $\Upsilon>2$ can be achieved in a waveguide-type system, where the reference pulse velocity can be made small compared to the group velocity of the information pulse by using different waveguide modes for the two pulses. Recording with $u_{a}>u_{b}$ (where both are signed quantities) provides the inversion of the pulse longitudinal profile, i.e., the compression factor $\Upsilon$ is negative in this case.

For $\Upsilon>1$, the original pulse length is larger than the depth of the "holographic plate". Thus, the front and the tail parts of the pulse do not need to remain inside the nonlinear medium at the same time while recording or retrieving is being performed. Thus, high compression of optical information within a hologram is achievable without compressing the input pulse itself.

Suppose now that a second short reference pulse $b_{\text {ret }}(\zeta)=B_{\text {ret }}\left(\zeta+u_{b} \Delta\right) \mathrm{e}^{\mathrm{i} \theta_{\text {ret }}}$ is injected into the medium in order to scan the hologram (Fig. 2). Using Eq. (2) and assuming constant reference pulse $B_{\text {ret }}$ and constant low-frequency wave $F_{0}$, the backscattered signal $a_{\text {out }}(\xi, \tau)=A_{\text {out }}\left(\xi-u_{a}(\tau-\Delta)\right)$ is precisely the original signal $A_{\text {in }}^{(0)}$ attenuated and delayed by time $\Delta$ :

$$
\begin{aligned}
& A_{\text {out }}\left(\zeta_{a}\right)=-\epsilon_{\text {rec }} \epsilon_{\text {ret }} A_{\text {in }}^{(0)}\left(\zeta_{a}\right) \mathrm{e}^{\mathrm{i} \theta_{\text {out }}}, \\
& \theta_{\text {out }}=\theta_{\text {ret }}-\theta_{\text {rec }}
\end{aligned}
$$

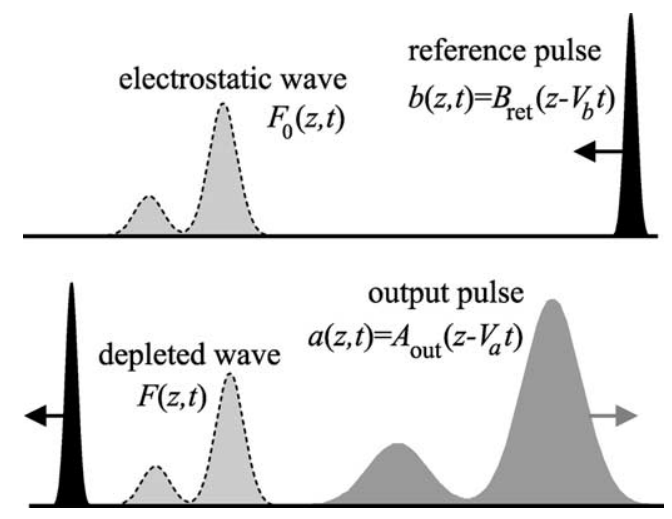

Fig. 2. Conceptual scheme of retrieving of 1D dynamic volume hologram from Raman media. While scanning with a reference pulse $B_{\text {ret }}$, the structure of the electrostatic wave $F_{0}$ is copied into the output pulse $A_{\text {out }}\left(\zeta_{a}\right) \propto F_{0}\left(\Upsilon^{-1} \zeta_{a}\right)$. For counter-propagating optical pulses in boundless medium, the stretching factor is $\Upsilon \approx 2$.
The results given by Eqs. (15) and (16) contain the major idea of 1D linear DVH, namely the recording and the retrieving optical pulse information by means of a low-frequency slowly propagating wave $f$. It remains to show that the information can be recorded and retrieved with fidelity under the condition of finite power of the reference pulses. Consider a recording process with $\epsilon_{\text {rec }}$ not necessarily small compared to unit but with a reference pulse still treated as given. The effective area where the pulses $a$ and $f$ evolve due to the interaction with the reference pulse is limited by the length of $B_{\text {rec }}$. For $\sigma \ll \Lambda$, one can assume that the whole interaction region shrinks to the point $\xi \approx u_{b} \tau$. From Eqs. (13) applied to the area close to the pulse $B_{\text {rec }}$ localized at $\zeta \in(-\sigma,+\sigma), \sigma \rightarrow 0$, one gets the profiles of the information pulses:

$$
\begin{aligned}
& a(\zeta, \tau)=A_{\text {in }}^{(0)}\left(-\left(u_{a}-u_{b}\right) \tau\right) \cos (U / 2), \\
& f(\zeta, \tau)=-A_{\text {in }}^{(0)}\left(-\left(u_{a}-u_{b}\right) \tau\right) \sin (U / 2) .
\end{aligned}
$$

After the interaction is over $(\tau \rightarrow \infty)$, in the frames moving together with the pulses $a$ and $f$ correspondingly, the wave envelopes (17) are given by

$$
\begin{aligned}
& A_{\text {in }}\left(\zeta_{a}\right)=A_{\text {in }}^{(0)}\left(\zeta_{a}\right) \cos \epsilon_{\mathrm{rec}}, \\
& F_{0}\left(\zeta_{f}\right)=-\mathrm{e}^{-\mathrm{i} \theta_{\mathrm{rec}}} A_{\mathrm{in}}^{(0)}\left(\Upsilon \zeta_{f}\right) \sin \epsilon_{\mathrm{rec}},
\end{aligned}
$$

where $\tau$ in the argument of $A_{\text {in }}^{(0)}$ was replaced with $\xi / u_{b}$ according to the adopted quasistatic approximation.

The linear approximation (15) is obtained from Eq. (18) under the assumption of a weak reference pulse $\left(\epsilon_{\text {rec }} \ll 1\right)$, which is generally true for conventional holography, where the stored (and the retrieved) images are proportional to the amplitudes of the reference waves (with few exceptions see $[8,16])$. On the contrary, in DVH, as one can see from Eq. (18), an increase of a reference pulse amplitude $\left(\epsilon_{\text {rec }}, \epsilon_{\text {ret }} \gtrsim 1\right)$ does not result in poor quality of pulse recording (and retrieving). Though the information pulses may change significantly during the interaction process, only their final amplitudes (but not the shapes) depend on the amplitudes of the reference pulses.

The dynamics of the recording process for large $\epsilon_{\text {rec }}$ is illustrated on Fig. 3 (retrieving goes similarly). Though the profiles of the information pulses are distorted significantly during the 

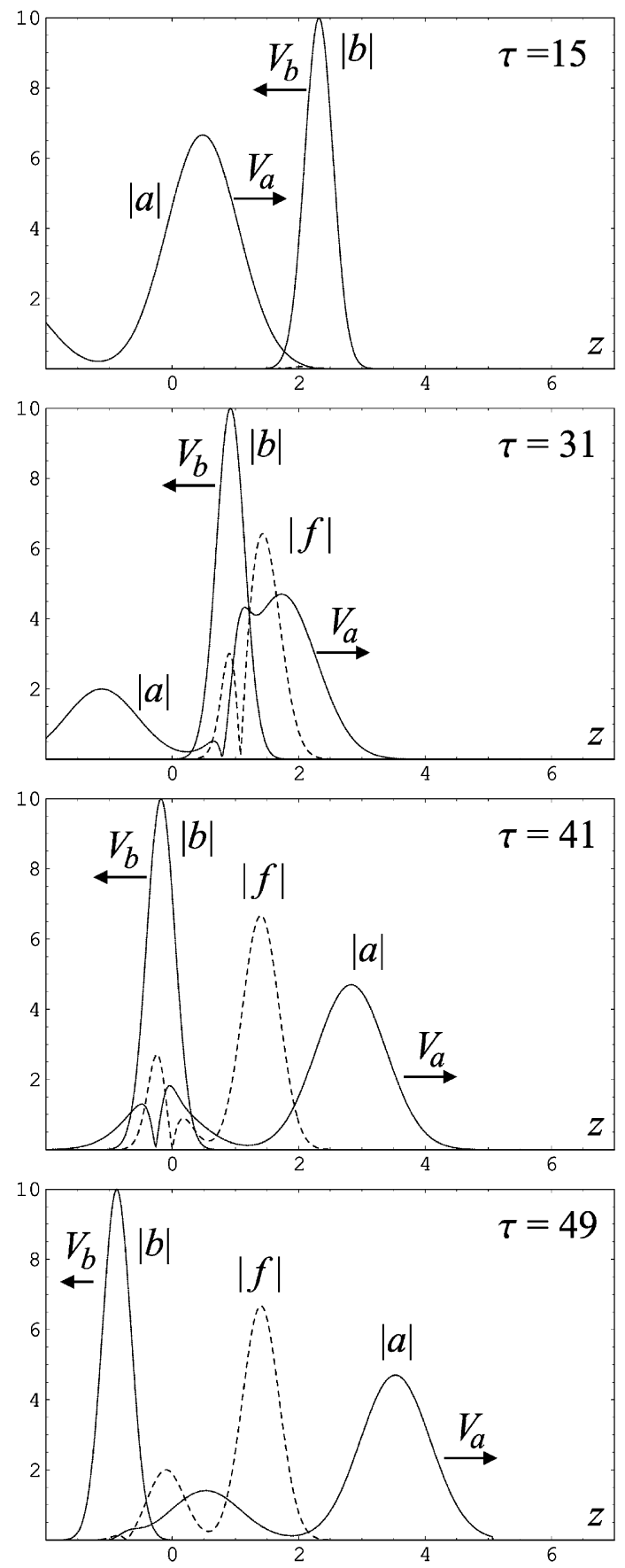

Fig. 3. Recording of the longitudinal structure of a 1D twohump optical pulse $a$ by a counter-propagating reference pulse $b$ into the profile of electrostatic wave amplitude $f$ (dashed); $V_{f}=0, \epsilon_{\mathrm{rec}}=1.3 \pi$, arbitrary units. interaction process, the shape of the optical pulse is imprinted into the low-frequency wave with fidelity if the length of the reference pulse is small compared to the length of the information pulse (see also Section 4).

The maximum amplitude of the recorded wave $F_{0}$ is achieved for $\epsilon_{\mathrm{rec}}=\pi(n+1 / 2)$, where $n$ is an integer. For this type of reference pulse, recording results in complete depletion of the input electromagnetic wave $A_{\text {in }}^{(0)}$, as follows from Eq. (18) (Fig. 4). This effect of depositing the energy of an optical pulse in a certain location might be useful for local heating of a nonlinear medium, since the energy of the hologram eventually transforms into the thermal energy of the medium, unless the hologram is retrieved beforehand. On the contrary, pulses with $\epsilon_{\mathrm{rec}}=\pi n$ result in transient appearance of the hologram $F_{0}$, which disappears after the interaction is over (Fig. 5).

Pulse retrieving is similar to recording. In order to retrieve the hologram stored in the wave envelope $F_{0}$ by a scanning pulse $B_{\text {ret }}$ (preserving its own shape during the interaction), one can apply Eqs. (13) with $N_{0}(\tau)=F_{0}\left(-\left(u_{f}-u_{b}\right) \tau\right) \mathrm{e}^{\mathrm{i} \theta_{\text {ret }}}$ and $\phi_{0}=$ $-\pi / 2$. The retrieved wave envelope and the remaining envelope of the low-frequency wave at $\tau \rightarrow \infty$ are then given by

$$
\begin{aligned}
& A_{\text {out }}\left(\zeta_{a}\right)=\mathrm{e}^{\mathrm{i} \theta_{\text {ret }}} F_{0}\left(\Upsilon^{-1} \zeta_{a}\right) \sin \epsilon_{\text {ret }}, \\
& F\left(\zeta_{f}\right)=F_{0}\left(\zeta_{f}\right) \cos \epsilon_{\text {ret }} .
\end{aligned}
$$

Expressing $F_{0}$ in terms of the original signal $A_{\text {in }}^{(0)}$, one gets a retrieved light pulse of the shape precisely equal to the shape of the original signal:

$$
\begin{aligned}
& A_{\text {out }}\left(\zeta_{a}\right)=\alpha A_{\text {in }}^{(0)}\left(\zeta_{a}\right), \\
& \alpha=-\mathrm{e}^{\mathrm{i} \theta_{\text {out }}} \sin \epsilon_{\text {rec }} \sin \epsilon_{\text {ret }},
\end{aligned}
$$

from where the linear approximation (20) is obtained if $\epsilon_{\text {rec }}, \epsilon_{\text {ret }} \ll 1$.

Like the recording procedure, pulse retrieving preserves the shape of the information pulse, providing an output signal with the same profile, $\Upsilon$-times-stretched in space. In principle, this straightforward way of pulse conversion on the "light acceleration" stage can be used for constructing electromagnetic pulses of the required shape. Assume one is able to design the hologram $F_{0}\left(\zeta_{f}\right)$ by some external means. Since the envelope 

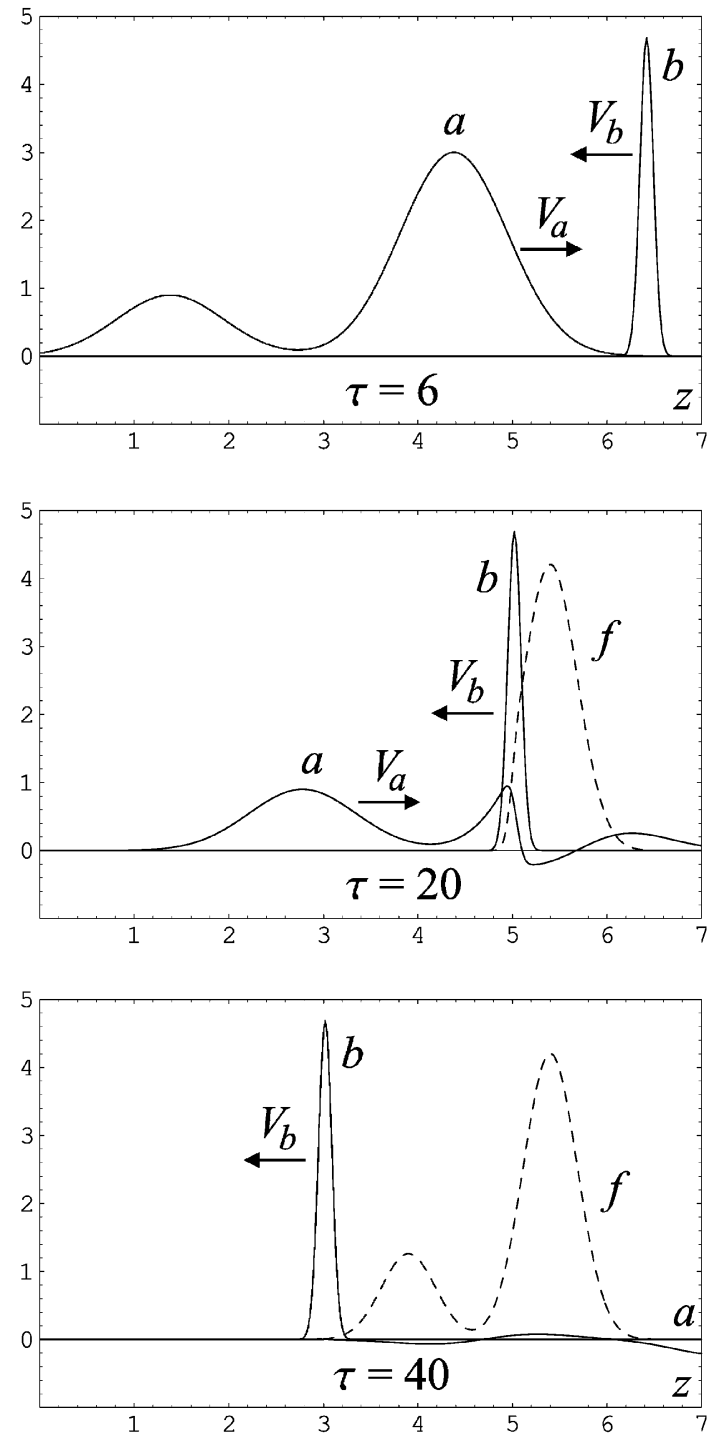

Fig. 4. Raman interaction between an optical pulse $a$ and a counter-propagating reference pulse $b$ with $\epsilon_{\text {rec }}=\pi / 2$ (arbitrary units). Electromagnetic pulse $a$ is almost fully depleted after the interaction; the electrostatic wave has a maximum possible amplitude.

$F_{0}$ does not propagate at high speed, the shape design for electrostatic oscillations might be easier to implement compared to the construction of a relatively short light pulse shape, while the light pulse is propagating. Further, by applying the retrieving procedure, this artificially designed pulse is accelerated up to the speed of light having the
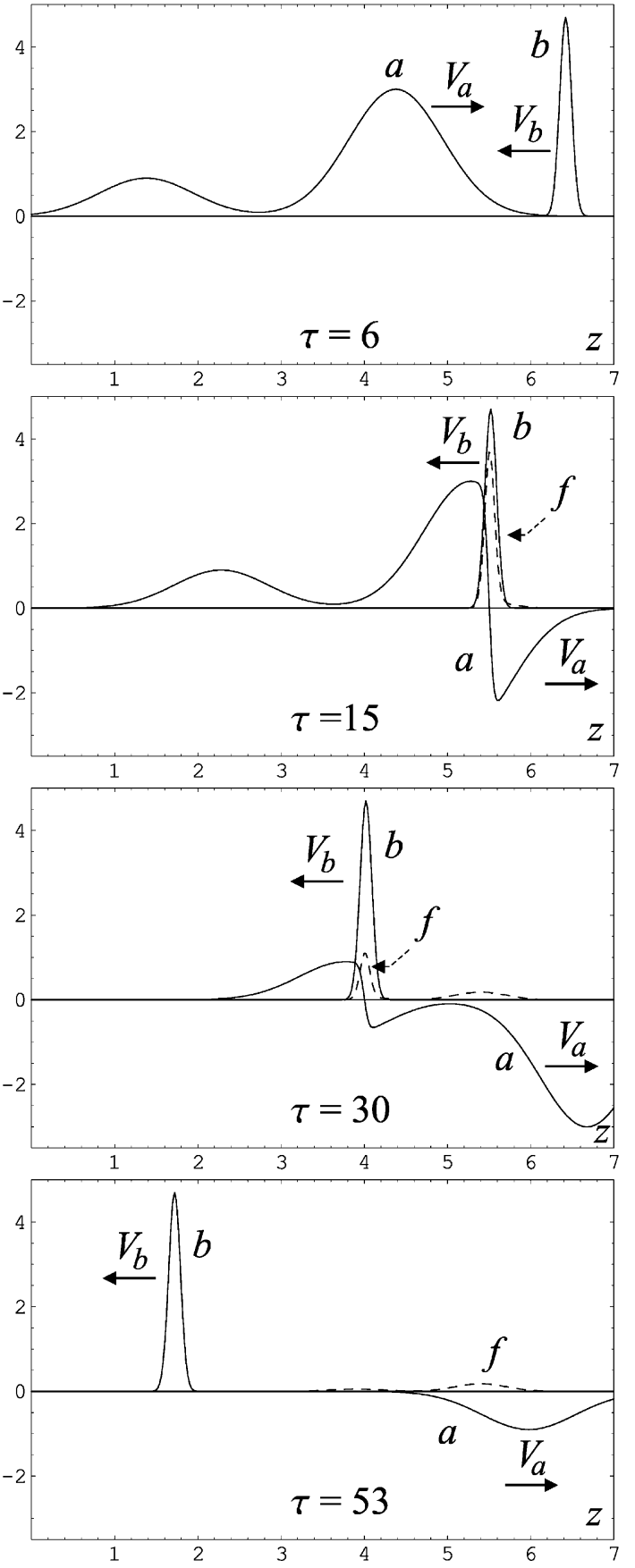

Fig. 5. Raman interaction between an optical pulse $a$ and a counter-propagating reference pulse $b$ with $\epsilon_{\text {rec }}=\pi$ (arbitrary units). No electrostatic wave $f$ (dashed) eventually survives after the interaction is over; optical pulse $a$ changes its polarity. 
required spatial profile $F_{0}\left(\Upsilon^{-1} \zeta_{a}\right)$, which might be useful for various applications where the laser pulse structure is critical.

As follows from Eqs. (19) and (20), the largest amplitude of the output signal $A_{\text {out }}$ is achieved with reference pulses having $\epsilon_{\text {ret }}=\pi(n+1 / 2)$, where $n$ is an integer. In this case, the hologram $F$ is "erased" during retrieving. Thus, reference pulses with $\epsilon_{\text {ret }}=\pi(n+1 / 2)$ essentially cool the medium by extracting the energy of electrostatic oscillations, which is converted into the energy of electromagnetic waves propagating away from the medium at the speed of light.

\section{Conditions of recording and retrieving optical information with fidelity}

To ensure that optical information can be recorded and retrieved with fidelity according to Eqs. (18) and (19), a certain number of conditions need to be satisfied. The first (geometric) requirement of a narrow reference pulse $(\sigma \ll \Lambda)$ provides that the image is "painted with a fine (small-scale) brush", so that high-spatial resolution of the hologram can be achieved. With a "coarse brush", small-scale details of the optical pulse are averaged out while being printed into the medium, so that only part of the information carried by the pulse is copied into the hologram. The same applies to the retrieving process, where fine details of the hologram cannot be retrieved with fidelity by means of a reference pulse, if its length exceeds the minimal longitudinal scale of the hologram.

In addition to the geometric constraint of narrow reference pulses, let us determine the limitations on pulses' amplitudes providing that the reference pulses can be treated as given during the interaction, as assumed in derivation of Eqs. (18) (20). The interaction between the optical pulse $A_{\text {in }}^{(0)}$ and the hologram $F_{0}$ being recorded provides a reference pulse with a tail $\delta B$ with duration of the order of $\Lambda$ :

$$
\begin{aligned}
& \delta B \sim \epsilon_{a} F_{0}^{*}, \\
& \epsilon_{a}=\int\left|A_{\text {in }}^{(0)}\left(\zeta_{a}\right)\right| \mathrm{d} \zeta_{a} \sim A_{\text {in }}^{(0)} \Lambda .
\end{aligned}
$$

(For clarity, in this section we take $u_{a} \sim u_{b}$ and suppose $\Lambda$ to be the length of both optical and electrostatic information waves, i.e., $\Upsilon \sim 1$ is assumed.) The interaction between $\delta B$ and $A_{\text {in }}^{(0)}$ results in hologram distortion $\delta F \sim \epsilon_{a} \delta B^{*}$. Therefore, the information can be recorded with fidelity $\left(\delta F / F_{0} \ll 1\right)$ only by weak information pulses:

$\epsilon_{a}^{2} \ll 1$.

In compliance with the assumptions made above, the strength of the reference pulse does not enter the condition of adequate recording.

In the presence of a finite-amplitude resonant electrostatic noise $F_{n} \ll F_{0}$, additional conditions must be taken into account to ensure the information is recorded with fidelity. While interacting with a reference pulse (Fig. 6), the noise $F_{n}$ gen-
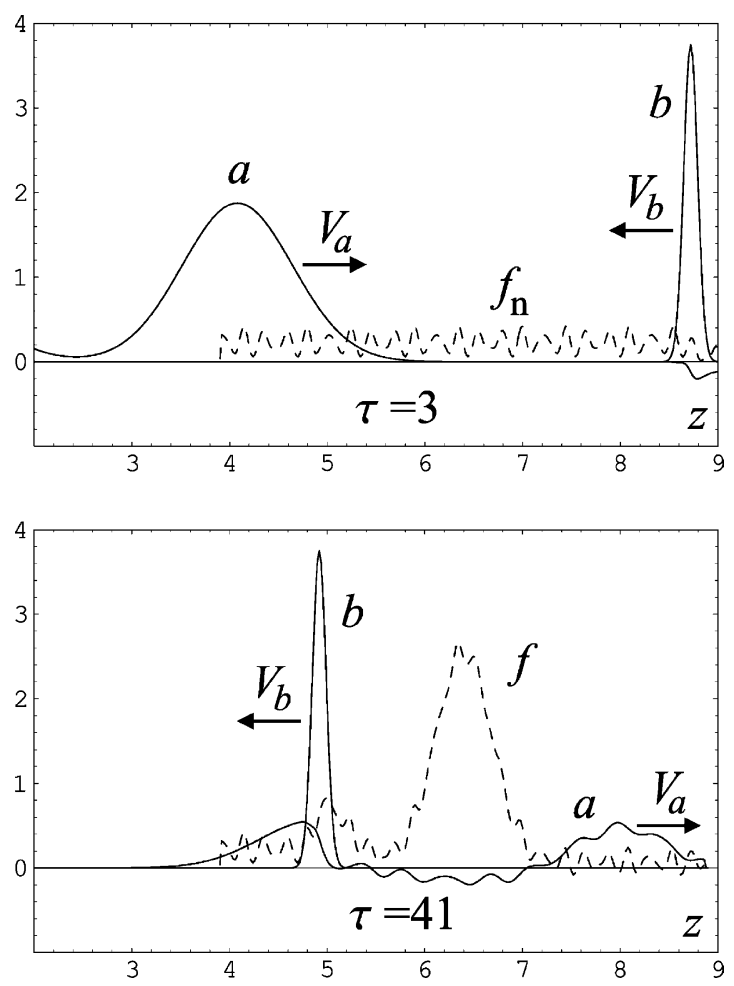

Fig. 6. Recording an optical pulse $a$ a into a noisy Raman medium by a counter-propagating reference pulse $b$ (arbitrary units). Thermal oscillations $f_{n}$ existing in the Raman medium are added to the desired profile of the electrostatic wave $f$ (dashed). 
erates a distortion of the input signal $(\delta A)_{n}^{(1)} \sim$ $\varepsilon_{\mathrm{rec}} F_{n}, \quad \varepsilon_{\mathrm{rec}}=\min \left\{\epsilon_{\mathrm{rec}}, 1\right\}$, which is immediately imprinted into the profile of the electrostatic wave $F$. This mechanism provides a relative distortion

$$
\begin{aligned}
& (\delta F)_{n}^{(1)} / F_{0} \sim \varepsilon_{\mathrm{rec}} \epsilon_{n 0} / \epsilon_{a}, \\
& \epsilon_{n 0}=\epsilon_{n} \Lambda / \Lambda_{n}, \\
& \epsilon_{n}=\int\left|F_{n}(z)\right| \mathrm{d} z \sim F_{n} \Lambda_{n}
\end{aligned}
$$

( $\epsilon_{\text {rec }} \neq \pi n$ is assumed), where $\Lambda_{n} \gtrsim \Lambda$ is the full path of a laser pulse inside the noisy medium. The other mechanism, through which $(\delta A)_{n}^{(1)}$ can influence the quality of recording is the distortion of the reference pulse provided by the interaction between $(\delta A)_{n}^{(1)}$ and $F_{n}$. Since $(\delta B)_{n}^{(1)} \sim \epsilon_{n}(\delta A)_{n}^{(1)}$, one gets for the corresponding deformation of the hologram $F_{0}$ :

$$
(\delta F)_{n}^{(2)} / F_{0} \sim \epsilon_{n} \epsilon_{n 0}
$$

In addition to this, the noise $F_{n}$ is also responsible for the direct distortion of the reference pulse $(\delta B)_{n}^{(2)} \sim \epsilon_{a} F_{n}$. While interacting with $A_{\text {in }}^{(0)},(\delta B)_{n}^{(2)}$ alters the electrostatic wave profile providing a distortion

$(\delta F)_{n}^{(3)} / F_{0} \sim \epsilon_{a} \epsilon_{n 0} / \varepsilon_{\mathrm{rec}}$.

Finally, the last mechanism of recording quality loss caused by the presence of $(\delta B)_{n}^{(2)}$ results from the evolution of the input signal: $(\delta A)_{n}^{(2)} \sim$ $\epsilon_{n}(\delta B)_{n}^{(2)}$, which, in turn, results in electrostatic wave profile distortion

$(\delta F)_{n}^{(4)} / F_{0} \sim \epsilon_{n} \epsilon_{n 0}$.

Neglecting higher-order corrections on $F_{n}$, one can estimate the total distortion of the recorded profile produced by the noise as

$$
(\delta F)_{n} / F_{0}=O\left(\varepsilon_{\mathrm{rec}} \epsilon_{n 0} / \epsilon_{a}\right)+O\left(\epsilon_{n} \epsilon_{n 0}\right)+O\left(\epsilon_{a} \epsilon_{n 0} / \varepsilon_{\mathrm{rec}}\right),
$$

from where it is seen that in noisy medium $\epsilon_{\mathrm{rec}} \sim \epsilon_{a}$ allows the best achievable quality of the hologram. For recording in the regime of $\epsilon_{\text {rec }} \sim 1$, which is useful for nonlinear information processing (see Section 6), one can summarize the validity conditions for quality recording as

$$
\epsilon_{n 0} \ll \epsilon_{a} \ll 1, \quad \epsilon_{n} \epsilon_{n 0} \ll 1 .
$$

The condition for retrieving information with fidelity, analogous to Eq. (22), can be obtained similarly and requires that the integral amplitude of the hologram is small:

$$
\begin{aligned}
\epsilon_{f}^{2} & \ll 1, \\
\epsilon_{f} & =\int\left|F_{0}\left(\zeta_{f}\right)\right| \mathrm{d} \zeta_{f} \sim F_{0} \Lambda .
\end{aligned}
$$

Thus, retrieving of the hologram, which was recorded in conformity with the condition (22), is always adequate, since $\epsilon_{f} \lesssim \epsilon_{a}$.

Taking the noise $F_{\mathrm{n}}$ into account, one is faced with the problem of generating optical signal $(\delta A)_{n}^{(1)}$ in addition to $A_{\text {out }}$ (Fig. 7):
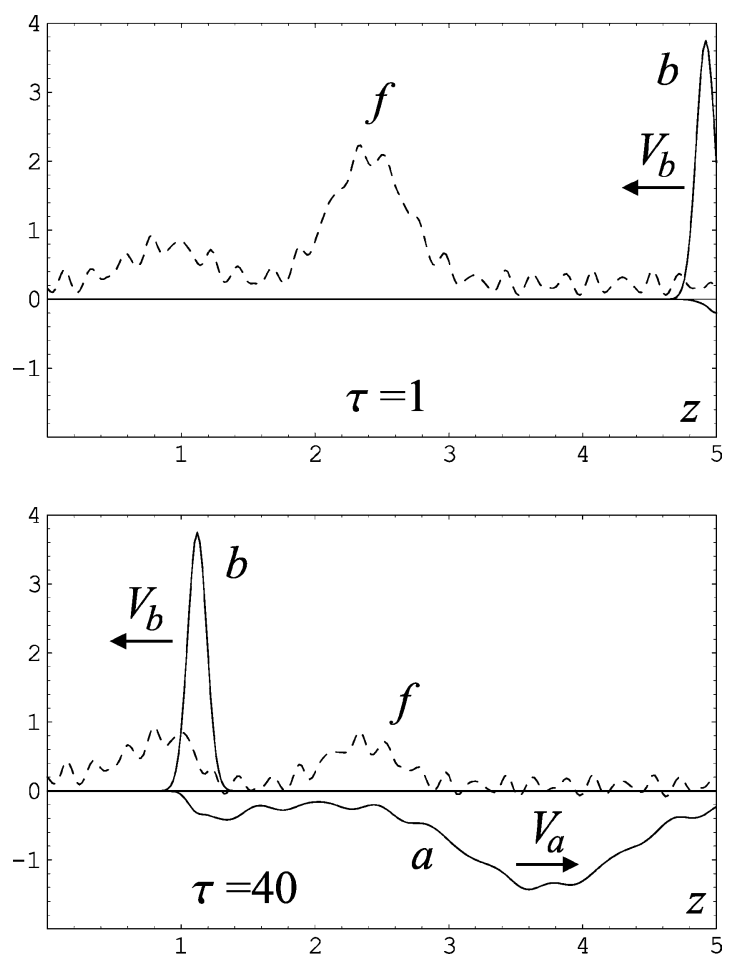

Fig. 7. Retrieving information from an electrostatic wave $f$ (dashed): an optical pulse $a$ is generated as a result of interaction between the electrostatic wave $f$ (dashed) existing in a noisy medium and a counter-propagating reference pulse $b$ (arbitrary units). The profile of thermal oscillations of spatial scale not less than the length of the recording pulse is copied into the output pulse; smaller-scale fluctuations are averaged out during the retrieving process and thus are not reproduced in the output pulse shape. 
$(\delta A)_{n}^{(1)} / A_{\text {out }} \sim \epsilon_{n 0} / \epsilon_{f}$.

On the other hand, the same distortion of the output pulse produces the distortion of the reference pulse $(\delta B)_{n} \sim \epsilon_{n}(\delta A)_{n}$, which, in turn, results in the output signal distortion:

$(\delta A)_{n}^{(2)} / A_{\text {out }} \sim \epsilon_{n} \epsilon_{n 0}$.

The higher corrections are of the order of $\epsilon_{n}^{2} \epsilon_{n 0} / \epsilon_{f}$, so that one needs to have $\epsilon_{n}^{2} \ll 1$ for them to remain small. Then the total distortion of the output signal by the noise $F_{n}$ is given by

$(\delta A)_{n} / A_{\text {out }}=O\left(\epsilon_{n 0} / \epsilon_{f}\right)+O\left(\epsilon_{n}^{2}\right)$,

which requires that $\epsilon_{\text {rec }}$ should not be too small, since $\epsilon_{f} \sim \varepsilon_{\mathrm{rec}} \epsilon_{a}$. Finally, the summarized conditions for quality retrieving can be put in the form

$\epsilon_{n 0} \ll \epsilon_{f} \ll 1, \quad \epsilon_{n}^{2} \ll 1$.

It is worth noting that during retrieving electrostatic fluctuations of spatial scale less than the length of the recording pulse are averaged out and thus are not reproduced in the output pulse shape (Fig. 7) because of finite resolution of the retrieving procedure.

\section{3D dynamic volume holograms: multiplexing}

Until this point, we considered recording and retrieving a 1D dynamic volume hologram. The 1D DVH represents a snapshot of the longitudinal structure of an optical pulse imprinted into some low-frequency slowly propagating wave. However, a similar technique is applicable for recording and retrieving also the transverse structure of $3 \mathrm{D}$ pulses.

First, consider the case when the width of the medium is small compared to the diffraction (Raleigh) length of optical pulses $z_{R} \sim k R^{2}$, where $R$ is their minimal characteristic transverse scale, so that the geometrical optics approximation can be applied. For paraxial propagation of the information and the reference optical pulses, a 1D hologram is formed on each geometrical ray of the information pulse. In this case, the transverse field distribution of the input signal enters the solution for the stored and retrieved signal only parametrically:

$$
\begin{aligned}
& F_{0}\left(\zeta_{f}, \boldsymbol{r}_{\perp}\right)=-A_{\mathrm{in}}^{(0)}\left(\Upsilon \zeta_{f}, \boldsymbol{r}_{\perp}\right) \mathrm{e}^{-\mathrm{i} \theta_{\mathrm{rec}}} \sin \epsilon_{\mathrm{rec}}, \\
& A_{\text {out }}\left(\zeta_{a}, \boldsymbol{r}_{\perp}\right)=\alpha A_{\mathrm{in}}^{(0)}\left(\zeta_{a}, \boldsymbol{r}_{\perp}\right) .
\end{aligned}
$$

If the reference pulses are also nonuniform in the transverse direction, then $\epsilon_{\text {rec }}, \epsilon_{\text {ret }}, \theta_{\text {rec }}, \theta_{\text {ret }}$, and $\alpha$ are functions of $\boldsymbol{r}_{\perp}$. By varying the amplitude and the phase distribution of the reference pulses, one can perform various operations on the structure of the information pulse while recording and retrieving the hologram (see Section 6).

Any modification of the information pulse structure can obviously include modification of the pulse wave front by adjusting the wave fronts of the reference pulses. For example, suppose that either the recording or retrieval pulses have transverse phase variation, such as focusing or defocusing wave fronts. The transverse phase advances can then be captured either in the plasma wave or in the retrieval process. The result is that the original data pulse upon retrieval can be brought to focus or magnified. Similarly, by varying the angle between the velocities of the information and the reference pulses (which corresponds to a reference pulse linear phase dependence on a transverse coordinate), one can change the direction of the output pulse propagation, for example, to make it enter a certain channel of an optical information processing system where the reference signal came out from.

In the opposite limit of strongly diffracting pulses, recording and retrieving of the transverse structure of pulses, in principle, are also possible. For example, consider a waveguide-type electrodynamic system channeling the pulses while they are interacting with each other. In a multi-mode waveguide, the information light pulse decays on a finite number of vector eigenwaves, each capable of carrying optical information in its longitudinal profile independently. As shown below, under certain assumptions, such optical information can also be extracted separately from different modes to be recorded into a set of uncoupled 1D holograms. Later these holograms can be retrieved back into the form of an optical signal on demand.

Consider the information wave envelope $\boldsymbol{a}$ and the field of a reference optical pulse $\boldsymbol{b}$ given in a form of series of the normalized vector eigenwaves $\psi_{n}$ of the waveguide: 
$\boldsymbol{a}=R \sum_{n} a_{n}(z, t) \psi_{n}\left(\boldsymbol{r}_{\perp}\right) \mathrm{e}^{\mathrm{i} h_{n}^{(a)} z}$,

$\boldsymbol{b}=R \sum_{m} b_{m}(z, t) \psi_{n}\left(\boldsymbol{r}_{\perp}\right) \mathrm{e}^{\mathrm{i} h_{m}^{(b)} z}$,

$\left\langle\psi_{m} \mid \psi_{n}\right\rangle=\delta_{m n}$,

where $R$ is the radius of the waveguide, which we include as a normalization factor to equalize the dimensions of $\boldsymbol{a}, \boldsymbol{b}$ and $a_{n}, b_{m} ; h_{n, m}^{(a, b)}$ are the longitudinal wavenumbers of the corresponding waveguide modes; scalar product denoted with angular brackets stand for integrating over the waveguide cross-section. The equations governing the evolution of the individual mode amplitudes $a_{n}, b_{m}$ can be put in the form [15]

$\partial_{t} a_{n}+V_{n}^{(a)} \partial_{z} a_{n}=\mu_{a} \sum_{m} b_{m} f_{n m}$,
$\partial_{t} b_{m}+V_{m}^{(b)} \partial_{z} b_{m}=-\mu_{b} \sum_{n} a_{n} f_{n m}^{*}$,

where $V_{n, m}^{(a, b)}$ are the group velocities of the corresponding modes. The quantities

$f_{n m}=\left\langle\psi_{n}|\varphi| \psi_{m}\right\rangle \mathrm{e}^{-\mathrm{i}\left(\omega_{a}-\omega_{b}\right) t+i\left(h_{m}^{(b)}-h_{n}^{(a)}\right) z}$

represent the transverse moments of the full electrostatic wave potential $\varphi$. Assuming that the interaction is efficient for resonant waves only, one can put the equation for $f_{n m}$ in the form

$\partial_{t} f_{n m}+V_{n m}^{(f)} \partial_{z} f_{n m}=-\mu_{f} C_{n m} a_{n} b_{m}^{*}$,
$C_{n m}=R^{2} \int \mathrm{d}^{2} r_{\perp}\left|\psi_{n}^{\dagger} \psi_{m}\right|^{2}$,

where $V_{n m}^{(f)}$ is the group velocity of the electrostatic wave corresponding to the carrier frequency $\omega_{a}-\omega_{b}$ and the wavenumber $h_{n}^{(a)}-h_{m}^{(b)}$; dimensionless coefficients $C_{n m}$ represent form-factors of the order of unit. For single-mode reference pulse, $b_{m}=\delta_{m s} b_{s}$, which does not evolve during threewave interaction (for details, see Section 4), the equations for the modes of the information optical pulse $a_{n}$ and the "modes" of the plasma wave $f_{n s}$ split up into separate couples of equations

$\partial_{t} a_{n}+V_{n}^{(a)} \partial_{z} a_{n}=\mu_{a} b_{s} f_{n s}$,

$\partial_{t} f_{n s}+V_{n s}^{(f)} \partial_{z} f_{n s}=-\mu_{f} C_{n s} a_{n} b_{s}^{*}$,

with each couple (39) representing a 1D scalar problem equivalent to the one described by Eqs. (2) and (4).
Eqs. (39) prove that each mode of the information optical pulse can exchange information with the only resonant harmonic of low-frequency wave and cannot mix with other waveguide modes even while being scanned by a reference pulse. Due to this, multiplex data storing and retrieving inside a waveguide becomes possible. That is, a waveguide can be used as a multiplex data bus storing optical information within spatially overlapped but still independent information channels.

To get higher level of multiplexing, one can use different carrier frequencies of optical signals, which is termed color holography (see, e.g., $[17,18])$. As long as the frequency gap between the individual channels remains large compared to the inverse characteristic temporal scales of information pulses evolution, the frequency channels do not mix with each other. Thus, more independent holograms can be stored at the same location within a Raman medium.

It is important to have in mind that the above discussion concerning the technique of storing holographic images in waveguides filled with cold plasma is, at the present stage, primarily of academic interest. A cold plasma is a delicate medium, where non-idealities impose severe restrictions on quality of holographic images and the maximal time of optical information storing (see Section 7). Therefore, the proposed waveguide technique may not work adequately in plasma, because the waveguide resonant modes interaction requires even more limitations in comparison to those in vacuum. The major problem consists of dealing with plasma in the long waveguides that are needed to assure that the Raleigh length remains small compared to the interaction length of the waveguide modes. For example, for $\lambda=1 \mu \mathrm{m}$ and the radius of the waveguide of $50 \mu \mathrm{m}$ (usual quasioptical capillary), the Raleigh length is of the order of one $\mathrm{cm}$. This means that, for the assumed parameters, the plasma column must be made as long as tens of centimeters for the selective properties of the waveguide to provide sharp holographic imaging. Increasing the size of the system requires longer information pulses, which would result in smaller relative time of optical information storing. The smaller storage times result from the 
limited lifetime of the hologram due to Coulomb collisions and Landau damping (Section 7), which means that as the pulse duration grows, the maximal time of storing the information divided by the duration of the pulse (which need to be of nanosecond scale for given parameters) decreases. In addition, since sustaining long uniform plasma columns is quite challenging, the nonlinear interactions (additional to those resulting from Raman scattering) of optical modes due the presence of plasma inhomogeneities may represent a significant problem for recording and retrieving information in waveguides with fidelity [19]. We hope, however, that these issues may be overcome, and that media with the necessary properties can be realized.

\section{Information processing by DVH}

Dynamic holography allows not only storing and reading optical information from a nonlinear medium, but also processing optical information in a number of ways while recording and retrieving light pulses (see, e.g., [6-8]). In addition to those, higher flexibility of DVH allows more advanced processing of optical signals. To show this, consider first the simplest case of 1D pulse recording at low intensities (processing during retrieving is analogous). Assume, for example, nonzero reference pulse to have a small average $\epsilon_{\text {rec. }}$ In the first-order approximation on $\sigma / \Lambda$, the pulse shape can then be treated as proportional to the first derivative of the delta-function, $B_{\text {rec }}(\tau)=\tilde{\epsilon}_{\text {rec }} \delta^{\prime}(\tau)$, if $\tilde{\epsilon}_{\text {rec }} \gg \epsilon_{\text {rec }} \Lambda$. Using Eq. (14), one gets for the recorded pulse after the interaction is over $(\tau \rightarrow \infty)$ :

$F_{0}\left(\zeta_{f}\right)=\left(u_{f}-u_{a}\right) \tilde{\epsilon}_{\mathrm{rec}}\left(A_{\mathrm{in}}^{(0)}\left(\Upsilon \zeta_{f}\right)\right)^{\prime}$,

where the derivative applies to the whole argument of $A_{\mathrm{in}}^{(0)}$. The hologram then contains a snapshot not of the actual pulse shape but of its first derivative (Fig. 8). Analogously, higher derivatives and the results of other linear conversions applied to the original signal profile can be imprinted into the low-frequency envelope structure by further varying the shape of the reference pulse. Thus, analog optical information can be processed in
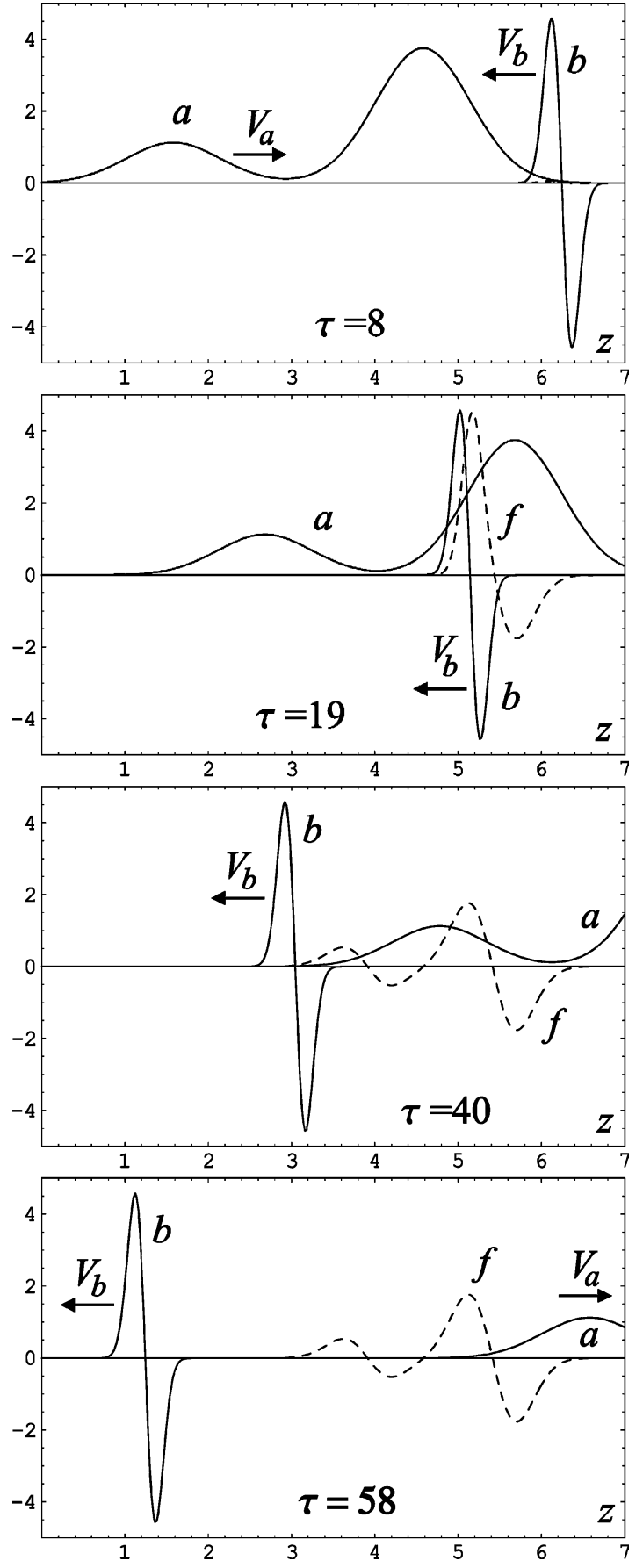

Fig. 8. Recording 1D pulse shape derivative: 1D two-hump optical pulse $a$ is differentiated by a counter-propagating reference pulse $b$ and the result is recorded into the profile of electrostatic wave amplitude $f$ (dashed); $V_{f}=0$, arbitrary units. 
various ways by means of linear DVH, which might be useful in applications of analog computing technology.

In addition to this, conventional Boolean operations can be performed on digital optical data. Consider 1D information pulse as 1D array of data bits, and the amplitude of the reference pulse as a single bit of information. At low power, the recording (or the retrieving) procedure provides multiplication of both signals, i.e., applies 'logical and' $(\wedge)$ to the pair of pulses: $F_{0}=A_{\text {in }}^{(0)} \wedge B_{\text {rec }}$. (Applying the same technique to $3 \mathrm{D}$ pulses, one can perform logical operations on 3D arrays simultaneously, which represents an additional advantage of DVH in comparison with the conventional dynamic holography, where only $2 \mathrm{D}$ arrays can be processed at the same time $[7,8]$.)

'Logical not' $(\neg)$ for optical pulses is also possible with nonlinear DVH, despite it represents a significant challenge for linear holography [7], where the amplitudes of the recorded and the retrieved signals are proportional to the amplitudes of the reference pulses. This operation can be performed by recording an identical unit into the hologram and scanning it with a data bit stored in the reference pulse with $\epsilon_{\mathrm{rec}}=\pi / 2$ (binary unit) or $\epsilon_{\text {ret }}=0$ (binary zero). What is left in the hologram is the "negative" of the information bit, i.e., $F=\neg B_{\text {rec. }}$ 'Logical or' ( $V$ ) can be performed similarly by retrieving the identical unit with two consequent scanning pulses $B_{\mathrm{ret}}^{(1,2)}$, both having $\epsilon_{\mathrm{rec}}=\pi / 2$ or $\epsilon_{\mathrm{ret}}=0$. In case when at least one of the two scanning pulses contains binary unit, the hologram will be erased, so that the remaining hologram bit contains 'negative logical or' applied to the pair of bits contained in the reference pulses: $F=\neg\left(B_{\text {ret }}^{(1)} \vee B_{\text {ret }}^{(2)}\right)$. In order to inverse the result, 'logical not' need to be applied. Other Boolean operations can be performed in similar ways.

The number of possible linear conversions, which can be performed on shapes of the optical pulses, can be increased further by taking advantage of $3 \mathrm{D}$ holography. By storing optical information in both longitudinal and transverse profiles of the wave envelopes, one can perform digital operations with 3D arrays of data or analogous operations with 3D continuous functions.

\section{DVH in nonideal media}

Within the approximation of the non-decaying low-frequency wave containing the holographic image, increasing the pulse storing time $\Delta$ does not result in loss of information. For practical implementations, however, it is necessary to find out how nonideal effects, which were not taken into consideration in the proposed model, may distort the hologram. Four basic ones can be identified: collisional and resonant damping of the low-frequency wave, background thermal fluctuations and transverse inhomogeneity of the Raman medium resulting in the wave front distortion of retrieved pulses. In this section, we will discuss these effects in application to a number of regimes of DVH in a cold plasma.

In plasmas, DVH can most easily be implemented on the base of laser pulses Raman backscattering on low-frequency Langmuir (plasma) waves with dispersion relation approximately given by $\omega_{f}^{2}=\omega_{p}^{2}$ [11]. In the cold plasma limit, namely at $k_{f} \lambda_{\mathrm{D}} \ll 1$, where $\lambda_{\mathrm{D}}=\sqrt{T_{\mathrm{e}} / 4 \pi n_{\mathrm{e}} e^{2}}$ is the Debye length (here $T_{\mathrm{e}}$ and $n_{\mathrm{e}}$ are the electron temperature and density; $e$ is the charge of electron), the group velocity of plasma waves are negligible. For now, let us also assume $\omega \equiv \omega_{a} \approx \omega_{b} \gg \omega_{p}$, so that the refraction coefficient for electromagnetic waves $n=1-\omega_{p}^{2} / \omega^{2}$ equals unit, and the dispersion relation for laser pulses is $\omega^{2}(k) \approx k^{2} c^{2}$, and $k \equiv k_{a} \approx-k_{b} \approx k_{f} / 2$.

Assuming weak collisional and resonant (Landau) damping of a plasma wave, one gets $v=$ $v_{C}+v_{L}$ for the resulting decay rate of the latter, where

$$
\begin{aligned}
& v_{\mathrm{C}}=\frac{4 \pi n_{\mathrm{e}} e^{4} \Lambda_{\mathrm{C}}}{m_{\mathrm{e}}^{1 / 2} T_{\mathrm{e}}^{3 / 2}}, \text { and } \\
& v_{\mathrm{L}}=\frac{1}{2} \sqrt{\frac{\pi}{2}} \frac{\omega_{p}}{\left(k_{f} \lambda_{\mathrm{D}}\right)^{3}} \exp \left(-\frac{1}{2\left(k_{f} \lambda_{\mathrm{D}}\right)^{2}}\right),
\end{aligned}
$$

are the partial decay rates corresponding to the two processes [20], where $\Lambda_{C} \sim 10$ is the Coulomb logarithm. We assume the ion charge $Z=1$ to 
provide the lowest possible decay. One can see that the requirements of low collision and Landau damping rates contradict one another. As the electron temperature grows, the collision rate decreases but Landau damping grows. From this point, choosing the optimal regime is a compromise, and for given electron density and laser wavelength $\lambda=2 \pi / k$, there always exists a upper limit for the time of pulse storing.

It is of interest to find the maximum storing time $\Delta_{\max }=v^{-1}$ measured in durations of the information pulse $\tau_{\text {in }}=N_{\text {in }} \tau_{\text {ref }}$, where $N_{\text {in }}=\Lambda / \sigma \gg 1$, and $\tau_{\text {ref }}=\sigma / c$ is the duration of each reference pulse:

$\frac{\Delta_{\max }}{\tau_{\text {in }}}=\frac{1}{N_{\text {in }} N_{\text {ref }}}\left(\frac{\omega_{p}}{v}\right)$.

Here $N_{\text {ref }}=\omega_{p} \tau_{\text {ref }}$ must be large compared to unit for the condition of resonant three-wave interaction to hold during recording and retrieving. For fixed $N_{\text {in }}, N_{\text {ref }}$ and given

$N_{D} \equiv n_{\mathrm{e}} \lambda^{3}=\pi \frac{\lambda}{r_{\mathrm{e}}}\left(\frac{\omega_{p}}{\omega}\right)^{2}, \quad \lambda=2 \pi / k$,

$r_{\mathrm{e}}=e^{2} / m_{\mathrm{e}} c^{2} \approx 2.82 \times 10^{-9} \mu \mathrm{m}$,

the optimal value of $N_{f}=\left(k_{f} \lambda_{D} \sqrt{2}\right)^{-1}$ can be found as the one, which minimizes the ratio

$\frac{v}{\omega_{p}}=32 \sqrt{2} \pi^{2} \Lambda_{\mathrm{C}} \frac{N_{f}^{3}}{N_{\mathrm{D}}}+N_{f}^{3} \sqrt{\pi} \exp \left(-N_{f}^{2}\right)$,

as follows from Eq. (41). Thus, the optimal conditions correspond to $N_{D}=A N_{f}^{-2} \exp \left(N_{f}^{2}\right), A \approx$ $48 \sqrt{2} \pi^{3 / 2} \Lambda_{C}$. Finally, the maximum possible delay limited by both collisional and collisionless damping is given by

$\frac{\Delta_{\max }}{\tau_{\text {in }}} \approx \frac{3}{2 A \sqrt{\pi}} \times \frac{N_{D}}{N_{f}^{3} N_{\text {in }} N_{\text {ref }}}$,
$N_{f}=\sqrt{\ln \left(N_{D} / A\right)+\ln \ln \left(N_{D} / A\right)}$,

and the optimum temperature is equal to

$T_{\mathrm{opt}} \approx \frac{m_{\mathrm{e}} c^{2}}{8 N_{f}^{2}}\left(\frac{\omega_{p}}{\omega}\right)^{2}$.

One might think that information can be stored with fidelity for times as large as desired by increasing $N_{D}$. However, in the proposed approach, $N_{D}$ is limited by the following factors. First, $\left(\omega_{p} / \omega\right)$ must remain smaller than unity to provide that the plasma density remains subcritical for laser pulses $(n>0)$. Also the maximum possible wavelength is limited by the size of the system, which must exceed the information pulse length. Thus,

$\lambda<2 \pi\left(\frac{\omega_{p}}{\omega}\right) \frac{L_{\text {plasma }}}{N_{\text {in }} N_{\text {ref }}}, \quad$ and

$N_{D}<\frac{\pi^{2}}{\sqrt{2}}\left(\frac{1}{N_{\text {in }} N_{\text {ref }}}\right)\left(\frac{L_{\text {plasma }}}{r_{\mathrm{e}}}\right)$

where we assumed $\left(\omega_{p} / \omega\right)_{\max }^{2}=1 / 2$, and $L_{\text {plasma }}$ is the length of the plasma layer to store the hologram in. From Eq. (48) it is seen that $N_{D}$ is limited by the length of the plasma layer, which is hard to maintain uniform (as required in the proposed model) on large distances.

In experiments, for storing information with fidelity, it is also necessary to make sure that the level of thermal fluctuations does not exceed the amplitude of the plasma wave itself. Assuming immobile ions, one has for the spectral density of electron density fluctuations $\left\langle n_{\mathrm{e}}^{2}\right\rangle_{\boldsymbol{k}} \approx n_{\mathrm{e}} / 2 N_{f}^{2}$ (in case when ions are thermalized, one needs to take $N_{f}=1$ in this formula) [20]. Integrate $\left\langle n_{\mathrm{e}}^{2}\right\rangle_{\boldsymbol{k}}$ over $\boldsymbol{k}$ in the range $\Delta k_{z} \sim \sigma^{-1}$ (fluctuations with wavenumbers larger than $\sigma^{-1}$ are averaged out while retrieving), $\Delta k_{\perp} \sim \lambda_{D}^{-1}$ (fluctuations with $\Delta k_{\perp}>\lambda_{D}^{-1}$ decay fast due to Landau damping) to get

$\delta \tilde{n}_{\mathrm{e}} \sim N_{f}^{-1} \sqrt{n_{\mathrm{e}} / \sigma \lambda_{D}^{2}}$

for the amplitude of density fluctuations resonant to the hologram profile. Regular density oscillations $\delta n_{\mathrm{e}} \sim k_{f} E_{f} / 4 \pi e$ in the field $E_{f} \sim\left(m_{\mathrm{e}} c / e\right) f \sqrt{\omega \omega_{p} / 2}$ of the plasma wave constituting the hologram [11] is given by

$\delta n_{\mathrm{e}} \sim\left(\lambda r_{\mathrm{e}} \sigma N_{\text {in }}\right)^{-1}$,

where we substitute the maximal possible dimensionless amplitude of the electrostatic field $f$ corresponding to $\epsilon_{f} \sim 1$ (see Section 4). As shown in Section 4, for adequate recording and retrieveing, it is necessary to have $\delta \tilde{n}_{\mathrm{e}} \ll \delta n_{\mathrm{e}}$ together with other conditions (28) and (33) satisfied.

In case if only the amplitude profile of the image is of interest (e.g., while processing digital data), the considered restrictions on the experimental parameters for DVH are sufficient. 
Table 1

Possible options for DVH experiment in a cold plasma at $N_{\text {in }}=\sigma / \Lambda=5, N_{\text {ref }}=\omega_{p} \tau_{\text {ref }}=20 \pi$

\begin{tabular}{lllll}
\hline Parameter & Exp. 1 & Exp. 2 & Exp. 3 & Exp. 4 \\
\hline Wavelength $\lambda, \mu \mathrm{m}$ & 10 & 400 & 10 & 800 \\
Electron density, cm ${ }^{-3}$ & $5 \times 10^{17}$ & $3 \times 10^{14}$ & $2 \times 10^{18}$ & $3 \times 10^{14}$ \\
Electron temperature, eV & 200 & 150 & 700 & 550 \\
Maximal possible time of pulse storing $\left(\Delta_{\max } / \tau_{\text {in }}\right)$ & 6.5 & 180 & 22.5 & 1200 \\
Pulse duration $\tau_{\text {in }}$, ps & 8.3 & 330 & 4.2 & 330 \\
Pulse length $\Lambda=c \tau_{\text {in }}, \mathrm{cm}$ & 0.25 & 10 & 0.13 & 10 \\
Information pulse maximal intensity, $\mathrm{W} / \mathrm{cm}^{2}$ & $4 \times 10^{10}$ & $2.5 \times 10^{7}$ & $8 \times 10^{10}$ & $1.3 \times 10^{7}$ \\
Reference pulses intensity, W/cm ${ }^{2}$ & $1 \times 10^{12}$ & $6.3 \times 10^{8}$ & $2 \times 10^{12}$ & $3.2 \times 10^{8}$ \\
$N_{f}=\left(\sqrt{2} k_{f} \lambda_{D}\right)^{-1}$ & 3.8 & 4.3 & 4.0 & 4.5 \\
$N_{D}=n_{\mathrm{e}} \lambda^{3}$ & $5 \times 10^{8}$ & $2 \times 10^{10}$ & $2 \times 10^{9}$ & $1.6 \times 10^{11}$ \\
\hline
\end{tabular}

Experiments 1 and 2 correspond to $\omega_{p} / \omega=0.2$, maximal possible wavelength for $L_{\text {plasma }}=10 \mathrm{~cm}$ is $\lambda=400 \mu \mathrm{m}$ (see Eq. (48)). For $\Delta \varphi<0.1 \mathrm{rad}$, one must have $\delta \bar{n}_{\mathrm{e}} / n_{\mathrm{e}}<0.003$ (see Eq. (51)). Experiments 3 and 4 correspond to $\omega_{p} / \omega=0.4$, maximal possible wavelength for $L_{\text {plasma }}=10 \mathrm{~cm}$ is $\lambda=800 \mu \mathrm{m}$. For $\Delta \varphi<0.1 \mathrm{rad}$, one must have $\delta \bar{n}_{\mathrm{e}} / n_{\mathrm{e}}<0.0016$. In all the four cases, $\delta \tilde{n}_{\mathrm{e}} \ll \delta n_{\mathrm{e}}$. The actual storing time can be several times longer because after the time $\Delta_{\max }=v^{-1}$ the amplitude of the hologram decreases only by a factor of $e \approx 2.7$.

However, for retrieving holographic images from nonuniform plasma preserving the phase fronts, there exists a limitation on the level of quasistatic plasma inhomogeneities. Because of the finite electron density perturbations, $\delta \bar{n}_{\mathrm{e}} / n_{\mathrm{e}}$, transverse to the laser beam, after the information pulse passes the distance equal to its own length, its phase front gets modulated with characteristic phase shift

$\Delta \varphi \sim \frac{N_{\text {in }} N_{\text {ref }}}{2}\left(\frac{\omega_{p}}{\omega}\right) \frac{\delta \bar{n}_{\mathrm{e}}}{n_{\mathrm{e}}}$,

which must remain small compared to unit for preserving the information about the phase. From this point, better quality of the hologram requires both small plasma density and small relative transverse variations of it.

As seen from Table 1, in a realistic temperature and frequency range, millimeter-size laser pulses can be stored in plasmas without significant distortion of their profiles on timescales long compared to the duration of the information pulses. For example, the information in a 8.3 ps long pulse of $10 \mu \mathrm{m}$ radiation can be stored in a plasma with density $5 \times 10^{17} \mathrm{~cm}^{-3}$ and length just $2.5 \mathrm{~mm}$. If the plasma is about $200 \mathrm{eV}$, then the information can be stored at least about 6 pulse durations, or for about 55 ps. Note that in fact the actual storing time can be several times longer because after the time $\Delta_{\max }=v^{-1}$ the amplitude of the hologram decreases only by a factor of $e \approx 2.7$. Unfortunately, in plasmas, restoring the phase front of the information pulses without distortion requires probably too homogeneous plasma comparing to what can be obtained in relatively simple experiments. For example, even for $N_{\text {in }}=1, N_{\text {ref }}=6 \pi$, $\omega_{p} / \omega=0.2$, one needs to have $\delta \bar{n}_{\mathrm{e}} / n_{\mathrm{e}}<5 \%$ to provide that $\Delta \varphi<0.1 \mathrm{rad}$.

It is worth mentioning that in addition to plasmas, there may be found other Raman media with low-frequency waves of a different nature, which would have even lower damping rates and allow storing holographic optical information for significantly longer time.

\section{Summary}

We generalize the conventional holography by combining the advantages of dynamic and volume holography into a single technique, which we designate by the term 'dynamic volume holography', or DVH. DVH allows capturing the information carried by a 3D laser pulse in the form of a slowly propagating low-frequency wave that persists for a time large compared with the pulse duration. If the low-frequency wave envelope (a dynamic volume hologram) is then probed with a short laser pulse, the stored information is retrieved in a second scattered electromagnetic wave. 
Both imprinting and scanning the hologram can conserve the optical information with fidelity. The effects of recording, storing and retrieving the light pulses can take place in arbitrary Raman media, such as plasmas, gases, fibers etc., when dissipation of the low-frequency wave (an information container) is negligible on time of storing the trapped signal. Possibly, the proposed technique could be useful for holography on the base of solid-state media at low temperatures where the dissipation of a dynamic hologram could be limited by quantum effects.

In addition to possible applications of dynamic volume holograms for designing 3D memory units, which might be especially useful for storing analog information, the proposed technique is also applicable for processing optical information. While storing or reading the pulse structure, the optical information can be processed as an analog or a digital signal, which allows simultaneous computing of 3D continuous or discrete arrays of data.

\section{Acknowledgements}

This work was supported by the US DOE, under contract DE-AC02-76 CHO3073, and Russian Foundation for Basic Research, grant 02-0206258.

\section{References}

[1] D. Gabor, Proc. R. Soc. A (London) 197 (1949) 454.

[2] Y.N. Denisyuk, Dokl. Akad. Nauk SSSR 144 (1962) 1275, in Russian.

[3] I.P. Woerdman, Opt. Commun. 2 (1970) 212.

[4] B.I. Stepanov, E.V. Ivakin, A.S. Rubanov, Dokl. Akad. Nauk SSSR 196 (1971) 567, in Russian.

[5] Y.N. Denisyuk, A. Andreoni, M.A.C. Potenza, Opt. Spectrosc. 89 (2000) 113.

[6] B.Y. Zeldovich, N.F. Pilipetskii, V.V. Shkunov, Wavefront Reversal, Nauka, Moscow, 1985, in Russian.

[7] A.A. Andreoni, M. Bondani, M.A.C. Potenza, Y.N. Denisyuk, E. Puddu, Rev. Sci. Inst. 72 (2001) 2525.

[8] A.I. Sokolovskaya, J. Russ. Laser Res. 19 (1998) 244.

[9] D.F. Phillips, A. Fleischhauer, A. Mair, R.L. Walsworth, M.D. Lukin, Phys. Rev. Lett. 86 (2001) 783.

[10] M. Fleischhauer, S. Yelin, M. Lukin, Opt. Commun. 179 (2000) 395.

[11] I.Y. Dodin, N.J. Fisch, Phys. Rev. Lett. 88 (2002) 165001.

[12] S. Mookherjea, A. Yariv, Phys. Rev. E 64 (2001) 066602.

[13] D.J. Kaup, A. Reiman, A. Bers, Rev. Mod. Phys. 51 (1979) 275.

[14] V.M. Malkin, G. Shvets, N.J. Fisch, Phys. Plasmas 7 (2000) 2232.

[15] I.Y. Dodin, G.M. Fraiman, V.M. Malkin, N.J. Fisch, Sov. Phys. JETP 95 (4) (2002) 625.

[16] A.V. Soroko, Opt. Spectrosc. 91 (2001) 446.

[17] L.A. Nefed'ev, V.V. Smartsev, Zh. Prikl. Spektrosk. 47 (1987) 640, in Russian.

[18] L.A. Nefed'ev, Opt. Spectrosc. 80 (1996) 125.

[19] M. Segev, A. Yariv, J. Shamir, Opt. Lett. 17 (1992) 1076.

[20] A. Akhiezer, I. Akhiezer, R. Polovin, A. Sitenko, K. Stepanov, Plasma Electrodynamics, Nauka, Moscow, 1974. 